\title{
Intravenous Thrombolysis for a Stroke Mimic Patient
}

\author{
Lilly Nguyen $^{a} \quad$ Joyce Hoonsuh Lee ${ }^{b} \quad$ Latha Gantic, d, e \\ Mark Rivera-Morales ${ }^{c, d}$ Larissa Dub ${ }^{c, d, e}$ \\ aTrinity Preparatory School, Winter Park, FL, USA; b Department of Biology, Brown University, \\ Providence, RI, USA; 'Department of Emergency Medicine, University of Central Florida,

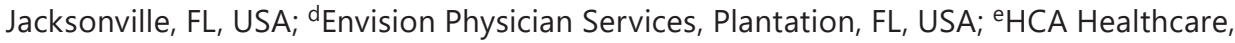 \\ Nashville, TN, USA
}

\section{Keywords}

Intravenous thrombolysis · Stroke mimics · Phentermine

\begin{abstract}
The authors present the case of a young woman on phentermine and herbal supplements who presented as an acute stroke alert with right-sided facial droop and numbness. She was treated acutely with intravenous tissue plasminogen activator (tPA). However, the workup did not reveal any evidence of cerebrovascular disease or cerebral infarct. The authors discuss plausible stroke mimics and the safety of administering tPA to such patients.
\end{abstract}

\section{Introduction}

Diagnosing stroke, a neurological deficit attributed to acute focal injury of the central nervous system, by vascular causes, can sometimes be convoluted. "Stroke mimics" refer to nonvascular conditions that present with acute neurological deficits of stroke, leading to false-positive stroke cases [1]. According to the National Institutes of Health (NIH), 9-31\% of stroke presentations can be attributed to stroke mimics [2] (Fig. 1). Stroke mimics can include complex migraines, seizures, blood pressure derangements, conversion disorder, adverse reactions to pharmacological agents, Bell's palsy, multiple sclerosis, sepsis, and lacunar syndromes not due to lacunar stroke [3]. 
Fig. 1. Infographic highlighting features of stroke mimics. Designed by Lilly Nguyen on canva.com.

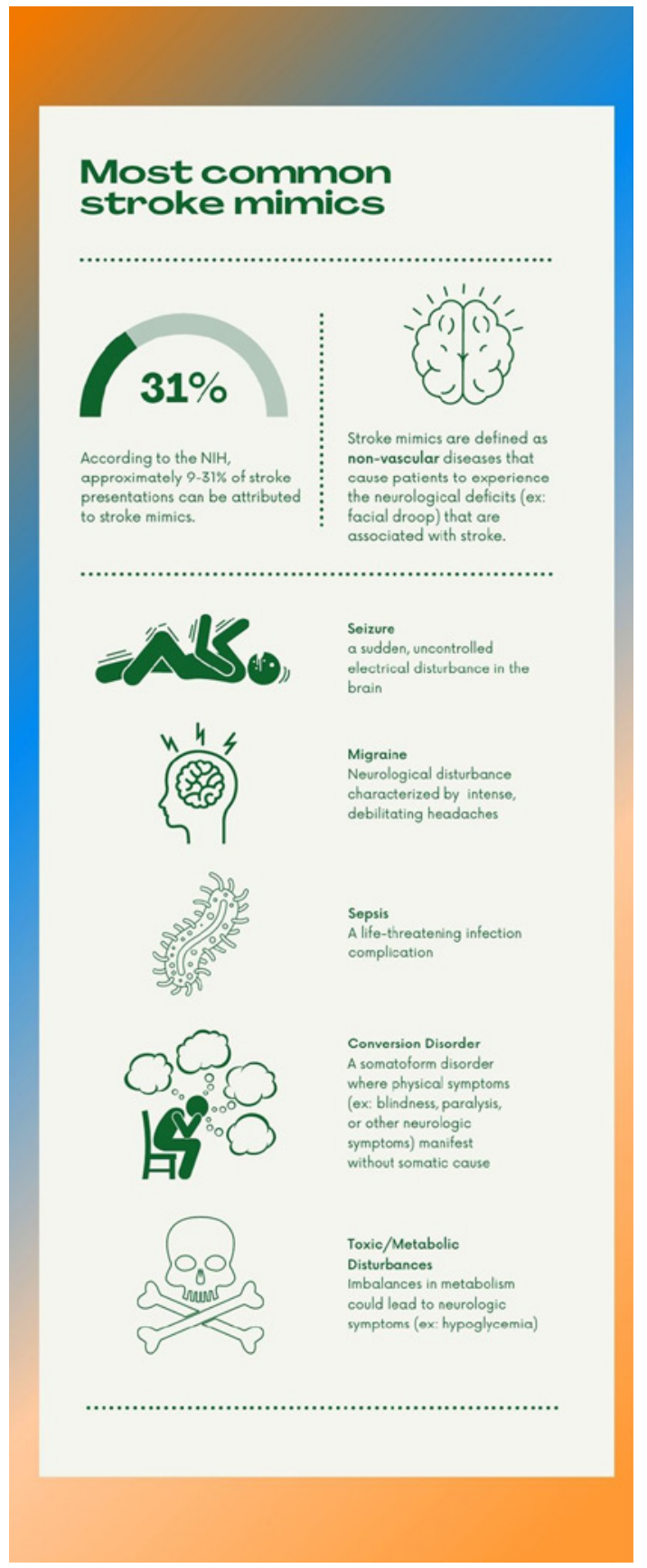

Tissue plasminogen activator (tPA) is the only FDA-approved drug for acute ischemic stroke (AIS). The safety and efficacy of intravenous (IV) tPA has been supported by an abundance of research in the decades following its approval for the treatment of AIS in 1996. Patients treated with IV tPA have a $30 \%$ greater likelihood of no or minor disability at 3 months if given within $3 \mathrm{~h}$ and $\sim 28 \%$ for those receiving within $4.5 \mathrm{~h}$ [4]. Indeed, every 15-min acceleration in the start of tPA after onset is associated with patients having 3-4\% greater odds of walking independently at discharge and being discharged to home rather than an institution and $4 \%$ lower odds of in-hospital death or hemorrhagic transformation of their infarct [5]. 
Nguyen et al.: Thrombolysis for Stroke Mimics

Because the outcomes of tPA treatment are highly correlated with the speed with which it is administered to AIS patients, the need for rapid recognition and tPA delivery increases the likelihood of tPA administration to stroke mimics [6]. Here, we report the case of a patient presenting with stroke-like symptoms and treated with tPA, with differential diagnoses including adverse reaction to supplements, complex migraines, and conversion disorder. The patient provided written informed consent for this case report.

\section{Case Report/Case Presentation}

A $24 \mathrm{y} / \mathrm{o}$ female with a history of hypertension and asthma presented via EMS as a stroke alert. She reported sitting at her computer and feeling like her face was drooping. Her right side felt weak and numb, and she had difficulty speaking. These symptoms lasted approximately $25 \mathrm{~min}$. She denied any changes in vision, headache, dizziness, nausea, vomiting, palpitations, or prior similar symptoms. She reported taking phentermine for weight loss for the past month. Her family history was significant for strokes and cardiac arrhythmia in her grandmother, hypertension in her mother, and diabetes in her father. She denied alcohol, cigarette smoking, or any illicit drug use.

EMS reported on scene a glucose of 58, and blood glucose on arrival was 83 . Her vital signs included a normal temperature, pulse, and respiratory rate. Her blood pressure was $150 / 83 \mathrm{~mm} \mathrm{Hg}$.

On physical examination, she was alert and awake. There were no signs of trauma. She had a normal heart and lung exam. Neurological examination revealed that she was able to follow 1-, 2-, and 3-step commands. Pupils were equal and reactive bilaterally. Extraocular movements were intact without any evidence of nystagmus. She had a right-sided facial droop with decreased sensation in the CN V distribution. There was no evidence of apraxia, extinction, neglect, or Gerstmann. Her speech did not reveal any aphasia. Speech was bradyphrenic with stuttering and dysarthria with buccolingual and guttural features. Hearing was normal on bedside testing, as was shoulder shrug and head turning. Tongue protrusion was normal in the midline. Motor examination revealed normal bulk, with reduced tone on the right. The right upper extremity showed 4-/5 strength in the fingers and wrist, 4/5 in the elbow, and $4+/ 5$ at the shoulder. The right lower extremity showed $3 / 5$ strength at the ankle, $4-/ 5$ at the knee, and 4/5 at the hip. Left side was 5/5 in both upper and lower extremities. There was no cogwheeling. Sensory testing revealed reduced pinprick in the right upper and lower extremities. Cerebellar testing revealed a normal finger to nose and heel to shin bilaterally. Reflexes were normoactive on the left and reduced on the right. Plantar response was flexor bilaterally, and no clonus was noted. Her National Institutes of Health stroke scale was 8.

Noncontrast head CT showed no acute abnormalities. CT angiography revealed patent vessels of the head and neck without evidence of significant stenosis, aneurysm, or vascular cutoff. CT perfusion study revealed symmetric cerebral perfusion, without evidence of core infarct or salvageable brain parenchyma.

Risks and benefits of IV tPA were reviewed with the patient, and she wished to be treated with the medication. No exclusion criteria for IV thrombolysis were present. She was given tPA within 80 min of symptom onset. She also received 1 dose of nicardipine due to her elevated blood pressure.

Laboratory analyses were unremarkable. The patient was admitted to the ICU as per post-TA protocol. Secondary stroke workup including MRI was unremarkable for any acute cerebrovascular event. The patient felt great the next day and was eager to go home. Her facial droop and sensory deficits had resolved.

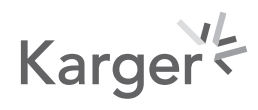


The neurology team concluded that the patient's symptoms could be due to the phentermine or herbal supplements the patient was taking or complex migraine or conversion disorder. She was instructed to stop taking phentermine and herbal supplements. Need for aspirin or statin therapy was not present. The patient was told she could pursue a hypercoagulable workup as an outpatient.

\section{Discussion/Conclusion}

This case aims to highlight the possible role that phentermine may play in causing strokelike symptoms in the stroke mimic patient and the lack of effect that tPA treatment has on stroke mimic patients. In this case study, a number of differential diagnoses were possible for the patient, including adverse reaction to supplements, complex migraines, and conversion disorder.

Adverse reaction to supplements: phentermine is one of the 5 weight loss supplements approved for use and is the most commonly prescribed weight loss supplement in the USA [7]. Phentermine triggers norepinephrine secretion and inhibits neural uptake, upregulating the noradrenergic and the dopaminergic central nervous system. These weight loss supplements have been linked to coronary vasospasm. Phentermine supplements may correlate with increased coronary sensitivity and predisposition to vasospasm [8]. The use of amphetamines and other anorexiants has been associated with ischemic stroke, myocardial infarction, and cardiac arrest. Phentermine, one such anorexiant with a chemical structure similar to amphetamines, has been linked to cerebrovascular disease as well as ischemic stroke [9].

Complex migraines: complex migraines are characterized by the presence of a migraine aura, including visual, sensory, or motor impairments. Complex migraines have also been generally associated with phentermine use. In a study, out of those who reported side effects of taking phentermine, only $0.02 \%$ reported experiencing migraine with aura. They were all female with an average age of 40-49 and some had high blood pressure. However, the association between phentermine use and experiencing a complex migraine was not strong enough to suggest a direct correlation [10].

Conversion disorder: conversion disorder was amongst the differential diagnoses for this patient although there was not much in the patient history to suggest psychological stress. Conversion disorder is a DSM-V diagnosis characterized as a somatoform disorder, presenting somatic symptoms with a psychological origin but no physiological abnormalities [11]. Studies suggest that symptoms are most common in young women [12]. Because there can be a range of presenting symptoms, conversion disorder presents with the symptoms of a multitude of disorders including multiple sclerosis, epilepsy, spine and motor diseases, myasthenia gravis, and stroke. The psychodynamic model of stroke conversion disorder attributes somatic symptoms to repressed emotional trauma. The cognitive-behavioral model, the model applicable to this case, suggests that exposure to information regarding symptoms of a certain disorder may later manifest when the patient actively and frequently looks for these symptoms [13].

The safety of tPA administration to stroke mimic patients has been studied in a variety of clinical studies [14] and found to be generally safe. For example, in a study of over 500 patients, $21 \%$ were ultimately found not to have had an ischemic stroke who had received tPA and none of them suffered symptomatic intracranial hemorrhage [15]. In a study of 621 patients of which 15 were stroke mimics, there were zero sICH. The most common symptom amongst stroke mimics was global aphasia without hemiparesis [16]. In a 2019 study of over 72,000 patients in the Get with Guidelines Stroke registry, a total of 2,540 patients who ultimately turned out to be stroke mimics received tPA. Their sICH rate was $0.4 \%$ compared with

\section{Karger'}


the $3.5 \%$ sICH rate in the group that actually suffered a cerebrovascular infarct [17]. These data support the safety of administering IV tPA to patients who otherwise meet criteria but end up ultimately having a stroke mimic rather than an actual cerebral ischemic event.

A variety of conditions can mimic AIS. Since time is of essence for tPA administration, it should not be withheld for fear of giving it to a potential stroke mimic, as these patients typically have an excellent prognosis.

\section{Acknowledgment}

This research was supported (in whole or in part) by HCA Healthcare and/or an HCA Healthcare affiliated entity. The views expressed in this publication represent those of the author(s) and do not necessarily represent the official views of HCA Healthcare or any of its affiliated entities.

\section{Statement of Ethics}

This study protocol was reviewed and approved by HCA Centralized Algorithms for Research Rules on IRB Exemptions (CARRIE)/IRB manager issued approval 2021-361. The patient provided written informed consent for publication of the details of the medical case and any accompanying images.

\section{Conflict of Interest Statement}

The authors have no conflicts of interest to declare.

\section{Funding Sources}

No funding was received for the preparation of the manuscript.

\section{Author Contributions}

L.N., J.H.L., and L.G. drafted the manuscript. M.R.M. and L.D. reviewed the case data and made revisions to the draft. All authors approved the final version of the manuscript.

\section{Data Availability Statement}

All data generated or analyzed during this study are included in this article. Further enquiries can be directed to the corresponding author.

\section{References}

1 Vilela P. Acute stroke differential diagnosis: stroke mimics. Eur J Radiol. 2017;96:133-44.

2 Hall MJ, Levant S, DeFrances CJ. Hospitalization for stroke in U S hospitals, 1989-2009. NCHS Data Brief. 2012; 95(95):1-8.

\section{Karger's}


3 Arboix A, Massons J, García-Eroles L, Targa C, Comes E, Parra O. Clinical predictors of lacunar syndrome not due to lacunar infarction. BMC Neurol. 2010;10:31.

4 National Institute of Neurological Disorders and Stroke rt-PA Stroke Study Group. Tissue plasminogen activator for acute ischemic stroke. N Engl J Med. 1995 Dec 14;333(24):1581-7.

5 Saver JL, Fonarow GC, Smith EE, Reeves MJ, Grau-Sepulveda MV, Pan W, et al. Time to treatment with intravenous tissue plasminogen activator and outcome from acute ischemic stroke. JAMA. 2013;309(23):2480-8.

6 Chapman SN, Mehndiratta P, Johansen MC, McMurry TL, Johnston KC, Southerland AM. Current perspectives on the use of intravenous recombinant tissue plasminogen activator (tPA) for treatment of acute ischemic stroke. Vasc Health Risk Manag. 2014;10:75-87.

7 Cohen JB, Gadde KM. Weight loss medications in the treatment of obesity and hypertension. Curr Hypertens Rep. 2019;21(2):16.

8 Prasad M, El Sabbagh A, Rihal C, Lerman A. Phentermine and coronary vasospasm-induced myocardial infarction. Mayo Clin Proc. 2019;94(7):1374-7.

9 Kokkinos J, Levine SR. Possible association of ischemic stroke with phentermine. Stroke. 1993;24(2):310-3.

10 Phentermine and Migraine with aura - a phase IV clinical study of FDA data. Accessed 2021 Aug 22. https:// www.ehealthme.com/ds/phentermine/migraine-with-aura/.

11 Orzechowski NM, Ytterberg SR. 37-year-old woman with headache. Mayo Clin Proc. 2006;81(10):1393-6.

12 Owens C, Dein S. Conversion disorder: the modern hysteria. Adv Psychiatr Treat. 2006;12(2):152-7.

13 Segal J, Lam A, Dubrey SW, Vasileiadis E. Stroke mimic: an interesting case of repetitive conversion disorder. BMJ Case Rep. 2012;2012:2.

14 Zinkstok SM, Engelter ST, Gensicke H, Lyrer PA, Ringleb PA, Artto V, et al. Safety of thrombolysis in stroke mimics: results from a multicenter cohort study. Stroke. 2013;44(4):1080-4.

15 Chernyshev OY, Martin-Schild S, Albright KC, Barreto A, Misra V, Acosta I, et al. Safety of tPA in stroke mimics and neuroimaging-negative cerebral ischemia. Neurology. 2010 Apr 27;74(17):1340-5.

16 Guillan M, Alonso-Canovas A, Gonzalez-Valcarcel J, Garcia Barragan N, Garcia Caldentey J, Hernandez-Medrano I, et al. Stroke mimics treated with thrombolysis: further evidence on safety and distinctive clinical features. Cerebrovasc Dis. 2012;34(2):115-20.

17 Ali-Ahmed F, Federspiel JJ, Liang L, Xu H, Sevilis T, Hernandez AF, et al. Intravenous tissue plasminogen activator in stroke mimics. Circ Cardiovasc Qual Outcomes. 2019 Aug;12(8):e005609. 